\title{
Forschung partizipativ und inklusiv gestalten? Ethische Reflexionen zu Interviews mit Kindern unter besonderer Berücksichtigung von Erwachsenheit
}

\author{
Katrin Velten · Julia Höke
}

Eingegangen: 30. Oktober 2020 / Angenommen: 7. März 2021 / Online publiziert: 12. Juli 2021

(C) Der/die Autor(en) 2021

Zusammenfassung Im Beitrag wird mit Fokus auf vier Ansprüche an die Erfassung von Kinderperspektiven der methodische Zugang des Interviews daraufhin reflektiert, wie im Kontext von kindlichem Eigensinn und generationaler Ordnung die Erwachsenheit der Forscher*innen aus forschungsethischer Sicht betrachtet werden kann und muss. In der sequenzanalytischen Re-Analyse von konflikthaften und weniger ergiebig erscheinenden Interviewsequenzen werden sog. Ad-hoc-Praktiken deutlich, in denen Forscher*innen in der Einmaligkeit der konkreten Interviewsituation Erwachsenheit und generationale Ordnung bei gleichzeitigem Interesse am Eigensinn des Kindes (re)produzieren. Diese sog. „Turning Points“ bieten Impulse für die Justierung des Begriffs der Angemessenheit und die Erweiterung von individuellen Forschungskompetenzen bei Kinderbefragungen.

Schlüsselwörter Partizipative und inklusive Forschung - Kinderinterviews · Forschungsethik · Erwachsenheit · Generationale Ordnung · Turning Point

Katrin Velten $(\bowtie)$

Universität Greifswald, Steinbeckerstr. 15, 17489 Greifswald, Deutschland

E-Mail: katrin.velten@uni-greifswald.de

Julia Höke

Katholische Hochschule NRW, Leostr. 19, 33098 Paderborn, Deutschland

E-Mail: j.hoeke@katho-nrw.de 


\title{
Participatory and inclusive research?-ethical reflexions about interviews with children with particular reference to adulthood
}

\begin{abstract}
In the paper, the methodological approach of the interview is reflected upon, with a focus on four demands on the recording of children's perspectives, how the researchers' adulthood can and must be considered from a research ethical point of view in the context of childlike attachments and generational order. In the sequence re-analysis of conflictual and less productive interview sequences, so-called ad hoc practices are particularly evident in which researchers (re)produce adulthood and generational order in the uniqueness of the concrete interview situation despite parallel interest in the child's obstinacy. These so-called "turning points" offer impulses for the adjustment of the concept of appropriateness and the expansion of individual research competences in child interviews.
\end{abstract}

Keywords Participatory and inclusive research · Interviews with children · Research ethics · Adulthood · Generational order · Turning point

\section{Einleitung}

Mit der Frage nach der Untersuchung von Partizipation und Inklusion in Kindertageseinrichtung und Grundschule geht die Herausforderung einher, wie es in der Forschung mit Kindern und über Kinder gelingen kann, sowohl die konkrete Umsetzung entsprechender Strukturen aus Perspektive der adressierten Kinder methodisch angemessen zu erfassen als auch gleichzeitig wesentlichen ethischen Anforderungen gerecht zu werden. Hierzu liegen seit längerer Zeit aus verschiedenen Forschungsrichtungen Ansätze vor, die Kinderperspektiven auf Partizipation und Inklusion qualitativ und quantitativ erfassen (World Vision e. V. 2018; Bosenius und Wedekind 2004; Hansen et al. 2011). Bei Studien, die die Kinder befragen, werden methodologische und ethische Fragen zur Angemessenheit z. B. hinsichtlich der methodischen Zugriffe im Sinn von Gütekriterien gestellt (Alderson und Morrow 2020; Bertram et al. 2016). Obwohl bereits in frühen Beiträgen neuerer Kindheitsforschung die Bedeutsamkeit von Erwachsenheit für die forschungsethische Reflexion der Angemessenheit sowohl von pädagogischen als auch forschenden Settings unterstrichen wurde (Honig et al. 1999), legen viele methodologische und ethische Auseinandersetzungen einen Schwerpunkt auf die Reflexion der Agency von Kindern und deren aktiver Rolle in der (Re)Produktion generationaler Ordnungssysteme sowie die Bearbeitung zugrunde liegender struktureller Rahmenbedingungen (Heinzel et al. 2012). Diese münden z. B. in Modelle oder Konzepte zur Anlage und Reflexion von Partizipation und Inklusion von Kindern in Bildungsinstitutionen (Alderson und Morrow 2020; Boban und Hinz 2009). Darüber hinaus wird diskutiert, inwieweit Forschung mit Kindern über Partizipation und Inklusion selbst partizipativ und inklusiv gestaltet werden kann bzw. muss. In den bisherigen Diskursen wird die Bedeutung von Erwachsenheit für die Angemessenheit der Handlungen pädagogisch tätiger und forschender Erwachsenen in den Praktiken partizipativ und inklusiv gestalteter Pädagogik und Forschung eher weniger betrachtet und reflektiert. Mit dem vorliegenden 
Beitrag wird sich diesem Anspruch genähert. Hierzu beschreiben wir das Zusammenspiel von vier Ansprüchen im Kontext der Befragung von Kindern, deren synchrone Bearbeitung/Erfüllung während konkreter Interviewsituationen zu Ad-hocPraktiken führen. In der Betrachtung solcher Ad-hoc-Praktiken wird deutlich, dass diese selbst Positionierungen zwischen Kindern und Erwachsenen hervorbringen. Eine kritische Reflexion der Erwachsenheit der Forscher*innen hat vor dem Hintergrund der Begründungslinien für die Erfassung von Kinderperspektiven einerseits und der Relation von Kind-Sein und Erwachsen-Sein andererseits eine hohe forschungsethische Relevanz für die Gestaltung inklusiver und partizipativer Forschung, da hierüber deutlich wird, wie herausfordernd die Erfüllung der Angemessenheit in der Forschung mit Kindern ist. Daran anschließend werden erste Konsequenzen für die Justierung des Begriffs der Angemessenheit in Kinderinterviews und Impulse für die Reflexion partizipativ und inklusiv gestalteter Forschungssettings im Sinn einer Erweiterung von Forschungskompetenzen formuliert.

\section{Erfassung von Kinderperspektiven: Begründungslinien}

Die Erfassung von Kinderperspektiven auf wesentliche gesellschaftliche Phänomene ist spätestens seit den frühen 1990er Jahren insbesondere in der internationalen Forschungslandschaft nicht wegzudenken. Im Kern geht es dabei zum einen sowohl um die Beschreibung grundlegender struktureller Unterscheidungs- und Marginalisierungsmechanismen und zum anderen um die adäquate Sichtbarmachung der Perspektiven von Kindern als Individuen sowie als gesellschaftliche Gruppe. Unter Einbeziehung der neueren soziologischen Kindheitsforschung bzw. „New Childhood Studies“ (Heinzel et al. 2012; Honig et al. 1999) wird das Kind nicht im Status des „Werdenden“, sondern als Person mit eigenen Fähigkeiten, Kompetenzen und Ressourcen gesehen.

Dementsprechend wird mit dem Ziel der Erfassung von Kinderperspektiven ebenso die Berücksichtigung der Kinderrechte angestrebt. Kinder haben das Recht auf Information, Verständnis, Stimme und Einflussnahme (Lundy 2007). Dieser Anspruch verschärft sich mit Blick auf die Institutionalisierung von Kindheit. Kinder verbringen heute immer mehr Zeit in pädagogischen Einrichtungen und damit in für sie geplanten und verzweckten Settings (Behnken und Zinnecker 2001). Kindertageseinrichtung und Grundschule werden zunehmend als Orte kindlichen Aufwachsens und Orte für Kinderkulturen gesehen und hinsichtlich ihrer Chancen und Grenzen bezüglich der Ermöglichung von Gestaltungsfreiräumen für Kinder betrachtet (Kasüschke 2016). Zudem gewinnen nicht zuletzt im Zuge der Ratifizierung der Behindertenrechtskonvention 2009 die Perspektiven marginalisierter Personen und Gruppen bei der Entwicklung und Ausgestaltung partizipativ und inklusiv gestalteter Settings in den Bildungsinstitutionen an Bedeutung. Hierbei ist die Partizipation aller Kinder bei gleichzeitiger Berücksichtigung besonders vulnerabler Gruppen oder Personen die Grundlage eines weiten Inklusionsverständnisses. Für Forschung, die sich für die Gestaltung der für Kinder relevanten partizipativen und inklusiven Lebensräumen und Settings interessiert, wird daher der Einbezug von Kindern und die Erfassung ihrer Perspektiven als zwingend notwendig betrachtet. 


\section{Zur Relation von Kind-Sein und Erwachsen-Sein}

Dem Anspruch, die Sicht der Kinder resp. den „,kindlichen Eigensinn“ zu erforschen, wird ein hoher Wert beigemessen: Kinder werden nicht nur als Datenlieferant*innen, sondern als Expert*innen ihrer Lebenswelt betrachtet (Wöhrer et al. 2017, S. 7). Diesem „Wert an sich“ liegt die Annahme zugrunde, dass Kinder selbst Agent*innen ihrer Bildungsbiografie und entsprechend handlungsfähig und -mächtig seien. Insbesondere aus kindheitssoziologischer Perspektive wird zum einen darauf hingewiesen, dass Agency nicht naturalistisch vorausgesetzt werden kann, sondern selbst konstruiert und mit bestimmten Positionierungen verbunden ist (Baader 2018). Honig et al. (1999) betonen zum anderen, dass die in der Eruierung von Kinderperspektiven hergestellte Differenz zwischen Kindern und Erwachsenen ebenso ein Konstrukt ist, das kritisch zu reflektieren ist. Ziel methodologischer Ansätze in der Kindheitsforschung ist daher, durch die Anerkennung des Kindes als „Experte seiner Lebenswelt“" die dem Forschungsprozess zugrundeliegende Machtposition zu relativieren und zugleich deren Nicht-Überwindung zu reflektieren (Fangmeyer und Mierendorff 2017, S. 10).

Im Anschluss daran beschäftigt sich der nationale Diskurs der letzten Jahre eingehend mit der Frage der aktiven Beteiligung des Kindes an der Reproduktion gesellschaftlicher Strukturen und generationaler Ordnung (Bühler-Niederberger 2011; Bühler-Niederberger et al. 2015). Das Konzept der generationalen Ordnung als Kernelement zur Beschreibung gesellschaftlicher Strukturen weist darauf hin, dass Kindheit sich in Relation zur Erwachsenheit konstituiert: Kinder befinden sich immer in Abhängigkeitsverhältnissen zu Erwachsenen. Bühler-Niederberger (2011) führt hierzu mit Blick auf die Allmacht der Erziehungssituation (Heinzel et al. 2012), die sich in der Interaktion von Kindern und Erwachsenen grundsätzlich zeigt, das Konzept des Handelns in kompetenter Gefügigkeit ein. Diesem liegt die Annahme zugrunde, dass Kinder immer vor dem Hintergrund eines von ihnen implizit oder explizit erwünschten Verhaltens agieren. In diesem Agieren erscheinen sie als ,soziale Alleskönner“, die das Abspüren von Regeln, das Begreifen von (divergenten) Anforderungen und ihr Funktionieren in diesen beherrschen (Bühler-Niederberger 2011, S. 225 ff.). Kritisch infrage zu stellen ist, inwiefern über kompetent gefügiges Handeln hinaus Freiräume für Kinder in sog. professionellen Interaktionen bestehen (ebd., S. 202ff.) und inwieweit die postulierte Angemessenheit überhaupt beurteilt werden kann, wenn das beobachtbare Agieren von Kindern bereits das Ergebnis vermuteter Erwartungen ist (ebd., S. 227).

Noch relativ unbeleuchtet ist das Konstrukt der Erwachsenheit und die Reflexion der eigenen Anteile an der (Re)Produktion generationaler Ordnung und ungleicher Machtverhältnisse (Fangmeyer und Mierendorff 2017, S. 12). In Bezug auf die Betrachtung der Relation von Kind-Sein resp. Kinderperspektiven und ErwachsenSein resp. Erwachsenenperspektiven sieht Hengst (2017) Potenzial im Deutungsansatz der ,differentiellen Zeitgenossenschaft“, in dem die Perspektiven von Kindern und Erwachsenen nicht per se diametral gegenübergestellt werden (ebd., S. 108). Unterschiede und Ungleichheiten zwischen Kindern und Erwachsenen werden so beleuchtet, jedoch nicht vorab über die Dimension der Generation festgeschrieben. Wie Kind-Sein und Erwachsen-Sein in konkreten Interaktionen verhandelt wird und 
welche Wirkungen dies in der Situation hat, zeigen Kelle und Möller-Schweda (2017) anhand einer Re-Analyse von beobachteten Gesprächen in Schuleingangsuntersuchungen: die Handlungs- und Gesprächsmuster der Agierenden sind von ihrem Status als Kind bzw. Erwachsener geprägt und bilden dementsprechend ritualisierte und habitualisierte Erwachsenen-Kind-Interaktion ab (ebd., S. 138). Anschließend an die Schuluntersuchungsgespräche, die weder als Forschungssetting noch als Interaktionssituation zur Erfassung von Kinderperspektiven i.e.S. angelegt waren, stellt sich die Frage, welche Ansprüche und damit einhergehenden Herausforderungen an das reflexive Handeln der erwachsenen Forscher*innen sich für Forschungssettings und Interaktionssituationen ergeben, die bewusst als Interviews zur Erfassung der Kinderperspektiven angelegt sind.

\section{Interviews mit Kindern: Ansprüche und Herausforderungen}

Mit Blick auf Rahmenbedingungen partizipativ und inklusiv angelegter Erhebungen von Kinderperspektiven bieten in den Childhood und Disability Studies entstandene Zugänge wie z.B. der Mosaic Approach (Clark und Moss 2011) oder alternative Befragungsformen wie z.B. das Walking Interview (Butler und Derret 2014) zwar mehr oder weniger weite Handlungsfreiräume für Kinder, ihre Perspektiven in einer Interviewsituation interaktiv zu vermitteln, sie heben die damit verbundenen Zuweisungen von Kindern als Befragte und Erwachsene als Fragende aber nicht auf: es ist anzunehmen, dass auch so angelegte Fragende*r-Befragte*r-Interaktionen in Interviews vor der Folie des Kind-Seins und Erwachsen-Seins ähnlich wie pädagogische Interaktionen im Sinn von Erziehungssituationen stark habitualisiert und ritualisiert sind. Zudem werden Kinder bereits durch die rechtliche Abhängigkeit vom formalen Einverständnis der erwachsenen Erziehungsberechtigten vor dem Zugang und der Teilnahme an einer Befragung unabhängig von Freiräumen in der Befragungssituation als Kinder positioniert, auch wenn dem Einverständnis der Kinder normativ in der Forschung mehr Gewicht beigemessen wird.

In dieser Weise werden voraussichtlich auch im in der Kindheits- und Grundschulforschung verbreitet eingesetzten leitfadengestützten Kinderinterview hierarchische Ordnungssysteme reproduziert (Fuhs 2012). Insbesondere in der konkreten Interview-Interaktion werden eine solche (Re)Produktion generationaler Ordnung und Handlungsspielräume aller am Interview Beteiligten in den Handlungen sichtbar. Somit stellt die nur bis zu einem bestimmten Grad planbare Einmaligkeit der tatsächlichen Interview-Interaktion die erwachsenen Forschenden als Fragende vor forschungsethische Herausforderungen, weil sie sich mit besonderem Fokus auf die Erwachsenheit und eigener (Forschungs)Interessen und deren Einfluss auf die Gestaltung der konkreten Erhebungssituation vier synchronen Ansprüchen und deren Ad-hoc-Bearbeitung/Erfüllung stellen müssen (vgl. Abb. 1).

Erstens ist mit Blick auf Planung und Durchführung leitfadengestützter Interviews grundlegend, den ,kindlichen Eigensinn“ und die Impulse der Kinder anzuerkennen und wertzuschätzen. Dieser Anspruch führt dazu, dass Interessen der Kinder aufgegriffen und Aktivitäten ermöglicht/unterstützt werden. Zugleich existiert aber zweitens auch ein „Eigensinn der Forschenden“. Dieser geht über die Einhaltung 


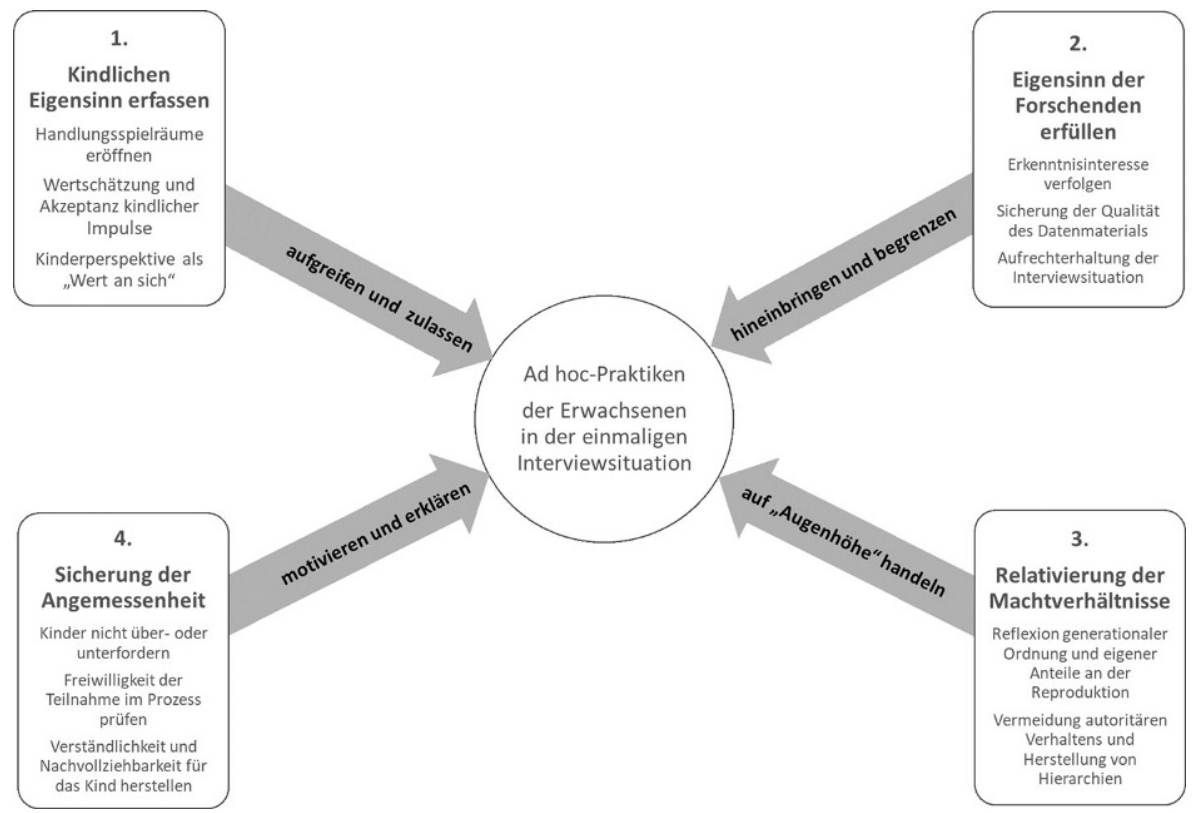

Abb. 1 Vier Ansprüche an die Ad-hoc-Praktiken Erwachsener in leitfadengestützten Befragungen von Kindern

gängiger Gütekriterien qualitativer Forschung wie z. B. die Vergleichbarkeit des Datenmaterials und intersubjektive Nachvollziehbarkeit der Analyse hinaus. Er richtet sich auf die Fokussierung eigener Forschungsfragen und zudem auf Vorstellungen darüber, was im Interview unter Abwägung der Aufmerksamkeitsspannen der Beteiligten und der zur Verfügung stehenden Gesamtzeit innerhalb des institutionellen Settings besprochen und gemacht werden soll. Beide Ansprüche sind auf der Planungsebene miteinander verknüpft, denn die sorgfältige Prüfung der Verständlichkeit handlungsleitender Begriffe und Leitfragen, die Ermöglichung vielfältiger, möglichst auch nonverbaler Ausdrucksformen und die Freiwilligkeit der Teilnahme sind besonders bei der Konzeption von Leitfadeninterviews mit Kindern zentral. In der Interviewsituation können sich beide Ansprüche allerdings auch diametral gegenüberstehen, wenn z. B. die Themen des Kindes auf ganz andere Inhalte abzielen als die eigenen Forschungsthemen und sich Forschende unmittelbar entscheiden müssen, wie weit/lang sie den Impulsen des Kindes folgen und wann eigene Themen mehr oder weniger deutlich platziert werden. Die Lösung stellt die Forschenden vor ein dilemmatisches Problem: „Welche Handlungsspielräume für den ,kindlichen Eigensinn" plane ich ein/lasse ich in der Situation zu, um die Impulse der Kinder anzuerkennen und wertzuschätzen?“ versus „Welche wesentlichen Interviewfragen/ Begriffe bringe ich ein und wie gestalte ich die Interviewsituation, um meine Forschungsziele zu erreichen?".

Drittens ergibt sich der Anspruch, die ungleichen Machtverhältnisse zwischen Befragten und Forschenden zumindest für die Zeit der Forschungssituation zu relativieren und die damit verbundenen Positionierungen von Kindern und Erwach- 
senen aufgrund ihrer Nicht-Auflösbarkeit gleichsam kritisch zu reflektieren. In der Forschungssituation geschieht dies z. B. über die Herstellung einer Beziehung, die sich normativ möglichst ablösen soll von zugewiesenen Verhältnissen und sich über das besondere Interesse der Forschenden an der Kinderperspektive konstituiert. Angestrebt wird eine „Begegnung auf Augenhöhe“ in dem Bewusstsein, dass diese tatsächlich nicht vorhanden sein kann. Viertens stellt der permanente Anspruch nach Angemessenheit der Situation die Forscher*innen vor die Herausforderung, unablässig zu prüfen, inwieweit insbesondere Freiwilligkeit und Verständlichkeit in der konkreten Interviewsituation (noch) gegeben sind, was zu weiteren Eingriffen und Steuerungen aus Erwachsenenperspektive führen kann (z. B. nachgeschobene Erklärungen, motivierende Kommentare, etc.).

In der konkreten Erhebungssituation im Leitfadeninterview mit Kindern vollziehen die Forscher*innen einen ständigen Balanceakt im Zusammentreffen der vier Ansprüche und ihrer Erfüllung. In diesem Balanceakt wird bereits angedeutet, dass die Verhältnisse innerhalb eines Interviews weniger klar zuordbar sind und im Interviewverlauf wechseln. Mit Blick auf die von Spriggs und Gillam (2017) über ein Studienreview identifizierte ethische Herausforderung, ,,competing interests [of researchers and children] and lack of reflexivity“ (ebd., S. 5, Einfügung KV/JH) in der Forschung zu Kinderperspektiven zu bearbeiten, könnten aus unserer Sicht insbesondere die Ad-hoc-Praktiken in der Interview-Interaktion für eine Reflexion bedeutsam sein. Im Anschluss an Kelle und Schweda-Möller (2017) stellen wir diese ins Zentrum der folgenden Re-Analyse.

\section{Anlage der Re-Analyse}

Mit Blick auf das aufgeworfene Desiderat gehen wir folgenden Fragen nach:

Wie werden Akteur*innenpositionen und Handlungsspielräume in Interviews mit Kindern ausgehandelt und wie werden Kindheit und Erwachsenheit in Adhoc-Praktiken von Forscher*innen innerhalb der Interviewsituation hervorgebracht?

Dem Beitrag liegen Interviewauszüge aus zwei Studien zugrunde, die jeweils die Perspektiven von Kindern auf die ihnen eröffneten Freiheitsgrade im Sinn von Partizipation in institutionellen Zusammenhängen erhoben haben. In der ersten Studie wurden im Juni und Juli 2016 Interviews mit 11 Erstklässler*innen in Anlehnung an das Lebensweltliche Interview nach Fuhs (2012) im Rahmen einer Schulbegleitforschung an einer partizipativ arbeitenden Grundschule erhoben (Höke 2020). Die insgesamt sieben Interviews fanden als Schulbegehung mit dem Auftrag an die Kinder statt, der Interviewerin ihre Schule mit Blick auf die „Orte, die aus ihrer Sicht bedeutsam sind“ zu zeigen. Das relativ offen angelegte Untersuchungsdesign, bei dem die Kinder sich z. B. aussuchen konnten, ob sie überhaupt, zu zweit oder allein teilnehmen und in welcher Reihenfolge welche Orte der Schule besucht werden, sollte dazu führen, den Kindern mehr Handlungsspielräume als in klassischen Interviewsettings zu eröffnen. Die Interviews wurden videografiert und anschließend basierend auf dem Gesprochenen transkribiert. 
In der zweiten Studie wurden im Zeitraum von März bis November 2013 in Anlehnung an Fuhs (2012) sowie den Mosaic Approach (Clark und Moss 2011) Interviews mit 22 fünf- bis siebenjährigen Kindern jeweils ca. vier Monate vor als auch nach Schulbeginn geführt (Velten 2019). Die Kinder wurden zu beiden Erhebungszeitpunkten gebeten, im Vorfeld der Interviews Fotos von den Handlungen bzw. Gelegenheiten zu machen, in denen sie sich partizipierend und kompetent fühlten. Die Fotos standen dann im Zentrum der Interviews. Alle Interviews wurden videografiert, um neben den verbalen die mimischen, gestischen und körpersprachlichen Ausdrucksmöglichkeiten der Kinder zu erfassen. Die Interviewtranskripte wurden vornehmlich auf Basis des Gesprochenen angefertigt. Ergänzungen zu Mimik, Gestik und Körpersprache wurden in eckigen Klammern transkribiert.

Basierend auf einer sequenziellen Analyse in Anlehnung an Schütz et al. (2012) sowie Kelle und Schweda-Möller (2017) werden für den Beitrag Interviewsequenzen unter Perspektive auf Kindheit und Erwachsenheit erneut analysiert. Ausgewählt wurden Situationen, die in der ursprünglichen Auswertung aufgrund vermeintlich misslingender kommunikativer Prozesse weniger ergiebig hinsichtlich der Forschungsfragen erschienen und deshalb nicht bzw. nicht vollständig berücksichtigt wurden. Dazu gehören vor allem Sequenzen, in denen beispielsweise eine so interpretierte Nicht-Beantwortung der Interviewfragen, Einbringen und Ignorieren von Themen oder Verweigerung und (drohender) Abbruch des Interviews sichtbar werden. Hiermit wurden insgesamt 10 Interviews ermittelt, die jeweils kritische Sequenzen beinhalten. Im Folgenden werden vier Sequenzen aus zwei Interviews exemplarisch dargestellt. Sie bieten u. E. vielfältige Reflexionsimpulse, sollen jedoch aufgrund des methodischen Zugangs und der Ausgangsgesamtheit der Daten keinesfalls generalisierend sein.

\section{Erkenntnisse der Re-Analyse: Entdeckte Ad-hoc-Praktiken und damit verbundene Positionierungen}

Unterschiedliche Erwartungen zur Gestaltung des Interviews werden in der ausgewählten ersten Sequenz aus Forschungsprojekt I deutlich, bei der die befragten Jungen auf die Frage, mit welchem Ort sie beginnen möchten, sofort den Sportplatz benennen und dann direkt ihren Ball holen. Die Erwartung der Kinder, sie könnten nicht nur über den Sportplatz erzählen, sondern auch dort spielen, wird von der Interviewerin im Dialog korrigiert:

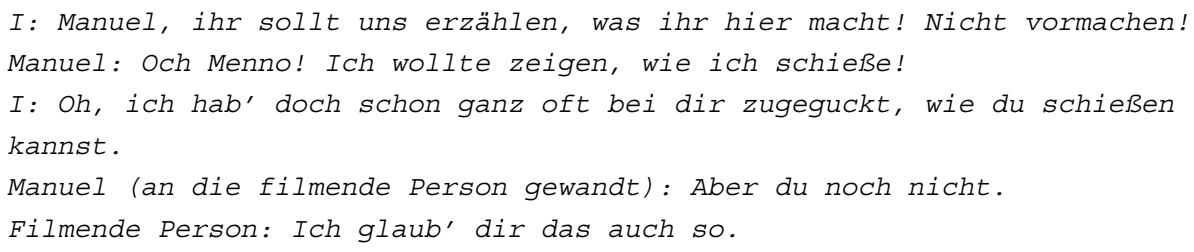

Deutlich wird hier ein Aushandlungsprozess innerhalb des Interviews, sprachlich markiert über die gegenseitigen Appelle ,ihr sollt“ und ,ich wollte“. Für die 
Jungen zeigt sich der kindliche Eigensinn in einer starken Verknüpfung zwischen Sprechen und Tun, vermutlich ist für sie nicht nachvollziehbar, wieso die Gruppe zum Sportplatz gehen soll, wenn dort nicht auch gespielt wird. Dies wird von der Interviewerin einerseits wertgeschätzt, ist der Impuls doch bereits eine Erkenntnis. Gleichzeitig besteht andererseits der Wunsch, die Erwartung der Kinder dahingehend zu korrigieren, dass die Interviewzeit dazu dienen könnte, jenseits des Unterrichts Spielzeit zu generieren. Zudem stellt sich aus Perspektive der Forscherin die Frage, inwieweit die Aufnahmequalität noch gegeben ist, wenn die Kinder während des Erzählens gleichzeitig Fußballspielen. Letztendlich nehmen die Jungen den Ball mit nach draußen und zeigen der Interviewerin und der filmenden Person tatsächlich ein paar Schüsse auf das leere Tor, währenddessen sie über die Spielsituationen auf dem Sportplatz erzählen.

Die folgende Interviewsequenz aus demselben Interview zeigt, was geschieht, wenn sich Aushandlungsprozesse in der Interview-Interaktion nicht über Kompromisse lösen. Das Interview dauert bereits über $40 \mathrm{~min}$ an, ohne dass die beteiligten Jungen mit der Interviewerin das Schulgebäude betreten haben. Die befragten Jungen haben die Interviewerin zu einem von ihnen als bedeutsam markierten Kletterbaum geführt und ihr, nachdem sie oben in den Zweigen sitzen, offenbart, dass dies verboten ist. Die Interviewerin reagiert darauf zunächst gelassen, was sich als Eröffnung von Handlungsspielräumen für die Kinder interpretieren lässt. Dass die Kinder trotz der Anwesenheit einer Erwachsenen etwas grundsätzlich Verbotenes tun, könnte zudem vor dem Hintergrund einer Relativierung des Machtverhältnisses gedeutet werden. Das Handlungsmuster der Interviewerin ändert sich allerdings, nachdem sie das Verbot ggf. mit Blick auf Verletzungsrisiken oder mögliche durch Regelverstoß entstehende Konflikte mit dem Schulpersonal reflektiert:

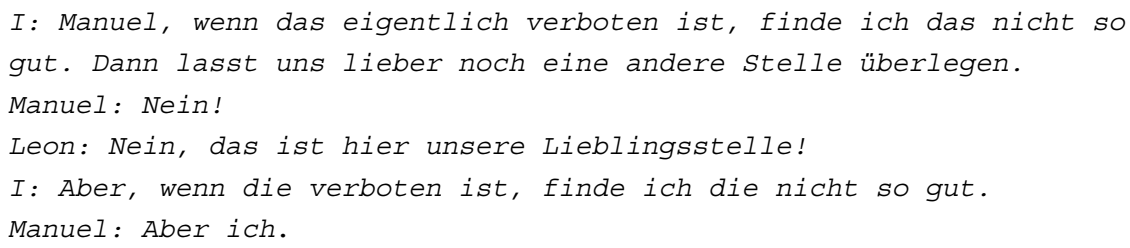

Die Interviewerin versucht zunächst, die Kinder zu einem Ortswechsel zu bewegen mit der Begründung, dass sie (als Erwachsene) diesen Regelübertritt ,nicht $g u t$ “ findet. Der Impuls erfolgt dabei über einen zwar im Sinn einer gemeinsamen Entscheidung verbalisierten, aber klaren Appell (,lasst uns“), ohne das (anscheinend relativierte) Machtverhältnis zwischen den Kindern und ihr aktiv wiederherzustellen. Ihr Interesse an einem Ortswechsel könnte neben einem möglichen Sicherheitsrisiko und Konflikten mit dem Schulpersonal auch vor dem Hintergrund des Erkenntnisinteresses und eigener Vorstellungen vom Verlauf des Interviews sowie der bisherigen Dauer und einem damit verbundenen Gedulds- und Aufmerksamkeitsverlust interpretiert werden. Als die Kinder dem Appell nicht Folge leisten, über sprachliche Markierungen (,nein“, ,aber ich“) sogar ein relativiertes Machtverhältnis von ihnen markiert wird, positioniert sich die Interviewerin als Erwachsene mit der Deu- 
tungshoheit über die Situation. Gegen die von ihr vorgenommene und nicht weiter begründete Positionierung wehren sich die Kinder deutlich:

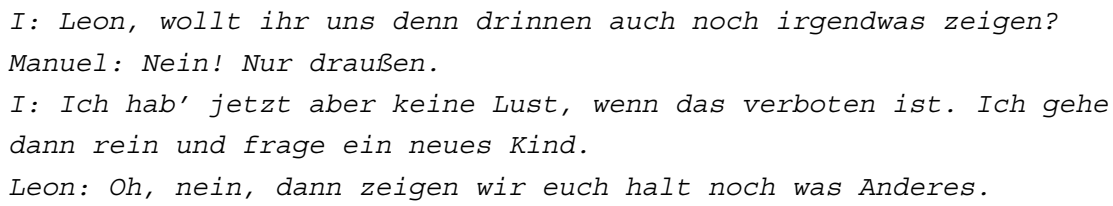

In der Aushandlung hinsichtlich des Ortswechsels wird nun klar, dass die Interviewerin Orte in der Schule aufsuchen möchte, die sie wahrscheinlich als enger mit dem Schulalltag verbunden vermutet, während die Jungen dagegen weiter draußen bleiben möchten. Die Ad-hoc-Reaktion der Interviewerin (, ich hab aber keine Lust“) bildet den „Turning Point“" innerhalb der Interview-Interaktion verbal ab: aus einer dem Eigensinn der Kinder zugewandten Haltung wird über eine WennDann-Konstruktion der Abbruch des Interviews angedroht. Die Interviewerin greift damit auf ein habitualisiertes bzw. ritualisiertes Handlungsmuster zurück, dass für Erwachsenen-Kind-Interaktionen gleichsam typisch wie pädagogisch problematisch erscheint, weil es eine - wenn auch vielleicht aus vermeintlicher situativ angenommener Alternativlosigkeit folgende - klare Machtausübung darstellt (Heinzel et al., 2012). Infolgedessen fügen sich beiden Jungen ad hoc in das erwünschte Verhalten (,Oh nein“) und sichern sich über einen Vorschlag kompetent die weitere Teilnahme am Interview.

In der nächsten beispielhaften Sequenz aus dem zweiten Forschungsprojekt wird die Hineinbringung des Themas durch die Interviewerin und die Nutzung einer entsprechenden Semantik deutlich. Das Interview findet in einem Raum in der Kindertageseinrichtung statt, der dem befragten Kind Alina durch ihre Teilnahme an verschiedenen Maßnahmen der Frühförderung bekannt ist. Die Fotos, die Alina zuvor in der Kindertageseinrichtung gemacht hatte, liegen auf einem Tisch ausgebreitet aus. Sie sieht ihre Fotos aufgrund des analogen Modus der genutzten Einmal-Kamera im Interview zum ersten Mal. Zum Einstieg betrachtet Alina ihre Fotos und kommentiert aus eigenem Impuls heraus einige Fotos hinsichtlich dessen, was und wer darauf zu sehen ist und wer diese Fotos gemacht hat. Währenddessen scheint Alina aufgeschlossen und motiviert, was über ihre im Transkript abgetragene Mimik und Gestik sowie Bewegungsaktivitäten angenommen werden kann. Sie spricht mit einer hellen Stimmfarbe und moduliert ihre Tonlage beim Sprechen. Eine Angemessenheit des Settings scheint gegeben. Daran anschließend bringt die Interviewerin folgende Aufforderung in das Interview:

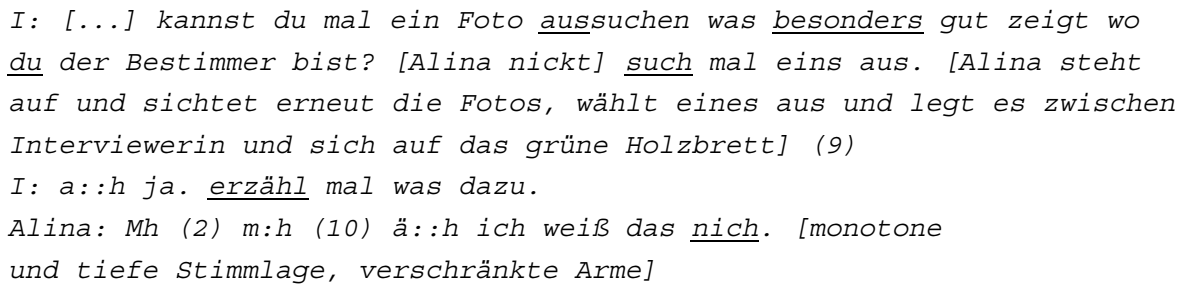


I: Was weißt du jetzt nich.

Alina: (2) Darüber weiß ich nich. [monotone und tiefe stimmlage, verschränkte Arme, Augenbrauen zusammengezogen, Kopf gesenkt]

I: Mhm du hast ja gerade das Foto ausgesucht äh weil das besonders zeigt dass du der Bestimmer. [Alina nickt] was bestimmst du denn da genau.

Alina: (3) Weiß ich nicht. [stützt ihren rechten Arm auf und verdeckt mit ihrer rechten Hand ihren Mund, auch beim Sprechen]

Das Hereintragen des eigenen Erkenntnisinteresses, von der Forscherin über das „Bestimmer*in sein“ verbalisiert, scheint nicht im Interesse Alinas zu liegen. Nachdem sie erst der Aufforderung nachkommt, entsteht im Vergleich zu den zuvor aus eigenem Impuls erfolgten kurzen Erzählfragmenten keine Narration. Im Gegenteil: ihre emotionale Stimmung scheint sich massiv zu verändern, was über ihre Stimmlage, Gestik und Körperhaltung angenommen werden kann. Das im Sinn des eigenen Erkenntnisinteresses hervorgebrachte wiederholte Nachfragen der Interviewerin scheint Alina zu irritieren, sie interpretiert es möglicherweise als eine Form der Wissensabfrage, wie ihre wiederholte Antwortformulierung andeutet (,Ich weiß das $\left.n i c h t^{*}\right)$. Damit zeigt sich auch diese Interview-Interaktion als eine klar hierarchische Erwachsenen-Kind-Interaktion im Sinn der Allmacht einer Erziehungssituation (Heinzel et al., 2012), die über das wiederholte Nachfragen manifestiert wird. Auch der Ad-hoc-Versuch der Interviewerin, an Alinas Auswahl des Bildes anzuknüpfen und so eine Balance im Sinn der Ansprüche wiederherzustellen, führt zu keiner Veränderung des Interaktionsprozesses. Im weiteren Verlauf reagiert Alina dann lediglich mit Kopfnicken bzw. -schütteln, so dass die Forscherin kurz darauf das Interview abbricht.

Bei der letzten Interviewsequenz handelt sich um den Abschluss des zweiten Interviewversuchs mit Alina, die nach dem Abbruch das Interview aus eigenem Impuls gemeinsam mit einer Freundin wiederaufnehmen möchte. Nach ca. $10 \mathrm{~min}$ des Interviews, in denen die Forschungsfragen der Forscherin im Fokus der InterviewInteraktion lagen, richten die beiden Mädchen ihr Interesse immer mehr auf die im Raum aufgehängten Plakate:

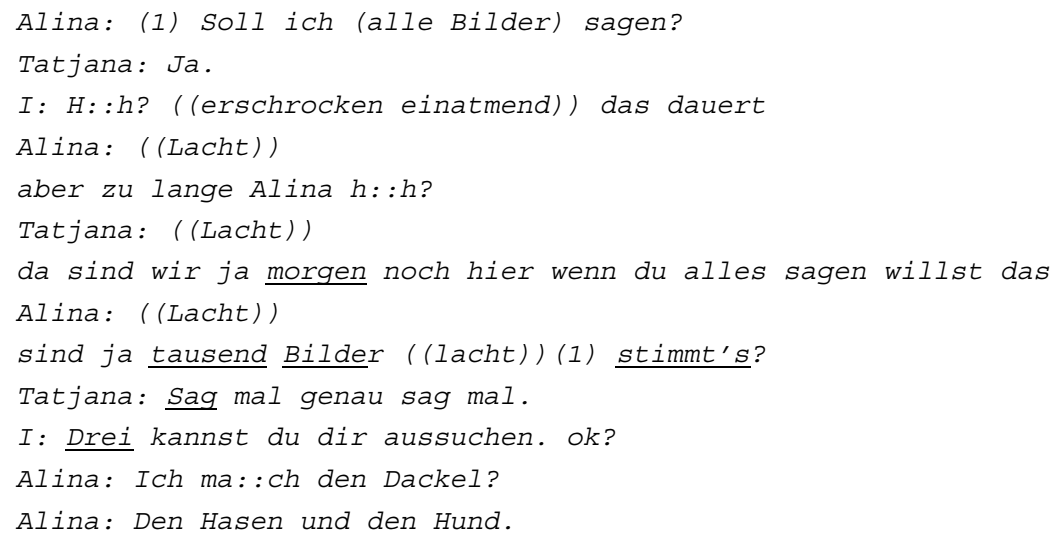




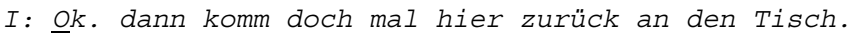

Alina: Da ist ein Dackel? da ist ein Hase? und da ist ein Hund.

Tatjana: Und das ist ein Leopard und das ist ein (1) (...) ok ok ok.

Alina: Sag alles Tatjana.

Tatjana: Das das das das da. Ein (...).

I : Stopp

Tatjana: (2) Ein Brötchen

I: Stopp

Tatjana: Ein Brötch: : :en (...)

I: Stopp ((lacht))

Alina: Ente Brötchen

Tatjana: Wassermelone: :n Banane Kiwi?

(Unverstanden: 25Sek.) [beide Kinder sind nicht mehr im Bildausschnitt $z u$ sehen, sie beschäftigen sich mit einer im Raum befindlichen

Sandkiste]

Alina und Tatjana beginnen, ihre eigenen Interessen in das Interview einzubringen: es entsteht die Idee, die auf einem Plakat abgebildeten Tiere zu benennen. Unter dem Anspruch der Wertschätzung des kindlichen Eigensinns (inhaltlich und zeitlich) durchaus möglich, wird Alinas Vorschlag von der Interviewerin mithilfe des rhetorischen Mittels der Übertreibung über die Stimmlage und Argumente (,tausend Bilder", ,morgen noch hier") als unmöglich gekennzeichnet, obwohl sie in diesem Moment gar nicht adressiert ist. Ihre Ablehnung begründet sie mit der Nicht-Passung ihrer Übertreibung des Spielvorschlags zu den Rahmenbedingungen des Interviews, also außerhalb ihrer Handlungsmächtigkeit. Dann schlägt sie eine verkürzte Variante (,drei kannst du dir aussuchen ok“) vor. Es wird innerhalb der hier deutlich hierarchisierenden Interviewerin-Kinder-Interaktion der Versuch der Interviewerin sichtbar, neben ihren eigenen dennoch zugleich den Eigeninteressen der Kinder gerecht zu werden. Zunächst entsteht zwar ein Aufsagespiel mit genau drei Bildern, das jedoch sogleich von den Mädchen umgedeutet wird. Auf das mit Nachdruck hervorgebrachte, jedoch aufgrund des Lachens als den Kindern grundsätzlich zugewandt interpretierbare Insistieren der Interviewerin (,Stopp“) entsteht ein Echo der Mädchen. Es scheint hier eine kurze Gleichzeitigkeit des hierarchisch orientierten Interviewerin-Kind-Verhältnisses und der situationsspezifisch eroberten Handlungsmacht der Kinder zu entstehen.

Im weiteren Verlauf dieser Sequenz nimmt die Interviewerin eine mehr und mehr beobachtende Haltung ein, worüber ein Handlungsspielraum entsteht, in dem die Mädchen die Interviewsituation zu ,ihrer“ Situation umdeuten: sie toben durch den Raum, spielen mit der Sandkiste, betätigen die Kamera. Im Hinblick auf das Zulassen des kindlichen Eigensinns und der Relativierung der Machtverhältnisse lässt sich das Handlungsmuster der Interviewerin als anerkennend und wertschätzend interpretieren. Jedoch lassen sich Alina und Tatjana auch durch wiederholte Angebote immer weniger dazu bewegen, sich auf eine Interaktion mit der Forscherin einzulassen. Infolgedessen führt diese dann den Abbruch der Situation herbei: 
I: (1) Alina mh dann müssen wir aufhören. dann geht es heute nich-

Alina: Ja.

I: Das merk ich grade.

Ähnlich wie in der zweiten Sequenz aus Forschungsprojekt I kommt es zum ad hoc auftretenden „Turning Point“ in der Interview-Interaktion, in der sich dasselbe habitualisierte Handlungsmuster der Interviewerin abbildet: die (Wenn-)DannKonstruktion, in der die Handlungen der Mädchen in direktem Zusammenhang mit erfolgenden Konsequenzen gebracht werden. Deutlich wird, dass die Freiraum gebende und eher beobachtende Interviewerin trotz alledem in „letzter Konsequenz“ diejenige ist, die Handlungsmacht in der Situation besitzt und ausübt. Ebenso vergleichbar mit den Handlungen der Jungen fügen sich auch die Mädchen als soziale Alleskönner (Bühler-Niederberger 2011, S. 225) kompetent der Abbruch-,,Regel““ der Interviewerin, allerdings ohne das Interview weiterzuführen.

\section{Konsequenzen und Ausblick}

In Kinderinterviews treffen basierend auf den im Beitrag fokussierten vier Ansprüchen im Kontext der Befragung von Kindern folgende Blickwinkel aufeinander: (1) Forschen mit Kindern zielt darauf ab, den „Eigensinn des Kindes“ unter Wahrung der Kinderrechte sichtbar zu machen mit dem Risiko, das Kind insbesondere im partizipativ und inklusiv gestalteten Forschungssetting auf besondere Weise als Kind, als be-fähigt/be-hindert zu positionieren und (un)bewusst Situationen zu forcieren, damit Kinder Aussagen tätigen, die sich von der ebenso konstruierten Erwachsenenperspektive deutlich abgrenzen lassen. (2) In Interviews zeigen sich divergente Erwartungen und Zielsetzungen zwischen Kindern und Interviewer*in, was in der Interviewzeit geschehen soll. Diese werden auf vielfältige Weise markiert und ausgehandelt und lassen sich unter der Perspektive der Herstellung von Kindheit und Erwachsenheit bzw. generationaler Ordnung reflektieren. (3) Der konkrete Ablauf der einmaligen Interviewsituation lässt sich vor dem Hintergrund des „Eigensinns der Forschenden“ im Zusammenspiel von Qualitätsansprüchen und Erkenntnisorientierung der Forschung und der Inszenierung von Machtverhältnissen re-analysieren, um Reproduktionen generationaler Ordnung aufzudecken.

Über diese Betrachtung stellt sich die immer wieder postulierte Angemessenheit bei der Erfassung von Kinderperspektiven als ein Balaceakt dar, der aus unserer Sicht insgesamt und vor allem mit Blick auf partizipativ und inklusiv gestaltete Forschungssettings theoretisch fundiert und forschungsethisch reflektiert werden müsste. In der Re-Analyse der ausgewählten Interviews werden verschiedene Ad-hocPraktiken der Interviewerinnen und befragten Kinder deutlich. Die Ad-hoc-Praktiken der Kinder lassen sich unter dem Konstrukt der sozialen Alleskönner (BühlerNiederberger 2011, S. 225) deuten, z. B. in Form des kompetenten Einlenkens in erwünschtes Verhalten oder im Verschließen/Verweigern. Dass Angemessenheit in Kinderbefragungen vor diesem Hintergrund aus dem Verhalten der Kinder schwer abzuleiten ist, wurde bereits problematisiert (ebd., S. 227). Mit Blick auf die erwachsenen Interviewerinnen werden verschiedene Ad-hoc-Praktiken deutlich, die 
zur Gestaltung von Aushandlungsprozessen angewendet werden, z. B. was im Interview thematisiert/de-thematisiert wird, wie lang es dauert und welche Zugänge den Interviewpartner*innen (nicht) zur Verfügung stehen. Diese Ad-hoc-Praktiken sind jedoch nicht nur Interaktionsstrategien, um den Balanceakt zwischen den vier Ansprüchen zu bearbeiten, sondern (re)produzieren selbst wieder Positionierungen zwischen den Interviewpartner*innen. Sie zeigen im Hinblick auf die generationale Ordnung und das Verhältnis von Kind-Sein und Erwachsen-Sein in der Re-Analyse einen von uns so bezeichneten „Turning Point“", in dem - z. T. auch plötzlich eine Veränderung/Rückkehr von einem möglicherweise situativ relativierten, am Forschungsinteresse orientierten zu einem manifesten hierarchischen, an Erwachsenheit orientierten Interaktionsverhältnis zutage tritt. In den exemplarisch analysierten Sequenzen wird dieses Umschlagen durch eine Wenn-Dann-Konstruktion verbal gerahmt.

Aus der Analyse lässt sich trotz der Limitierungen aufgrund der Exemplarität der Sequenzen annehmen, dass die reflexive Betrachtung insbesondere der ,Turning Points“ in den Interview-Interaktionen bedeutsam sein könnte, wenn es um eine Annäherung an die Angemessenheit in partizipativ und inklusiv angelegten Befragungen von Kindern gehen soll. Dabei spielen individuelle Reflexionsprozesse sicher in jedem Teilschritt des Forschungsprozesses eine wichtige Rolle, um jenseits grundsätzlicher methodologischer Überlegungen und ethischer Richtlinien für die partizipative und inklusive Forschung mit Kindern die Verantwortung der Forschenden und ihre individuellen Forschungskompetenzen in den Blick zu nehmen und (weiter) zu entwickeln. Ein zentrales Ziel wäre u. E. aber besonders, sich neben der

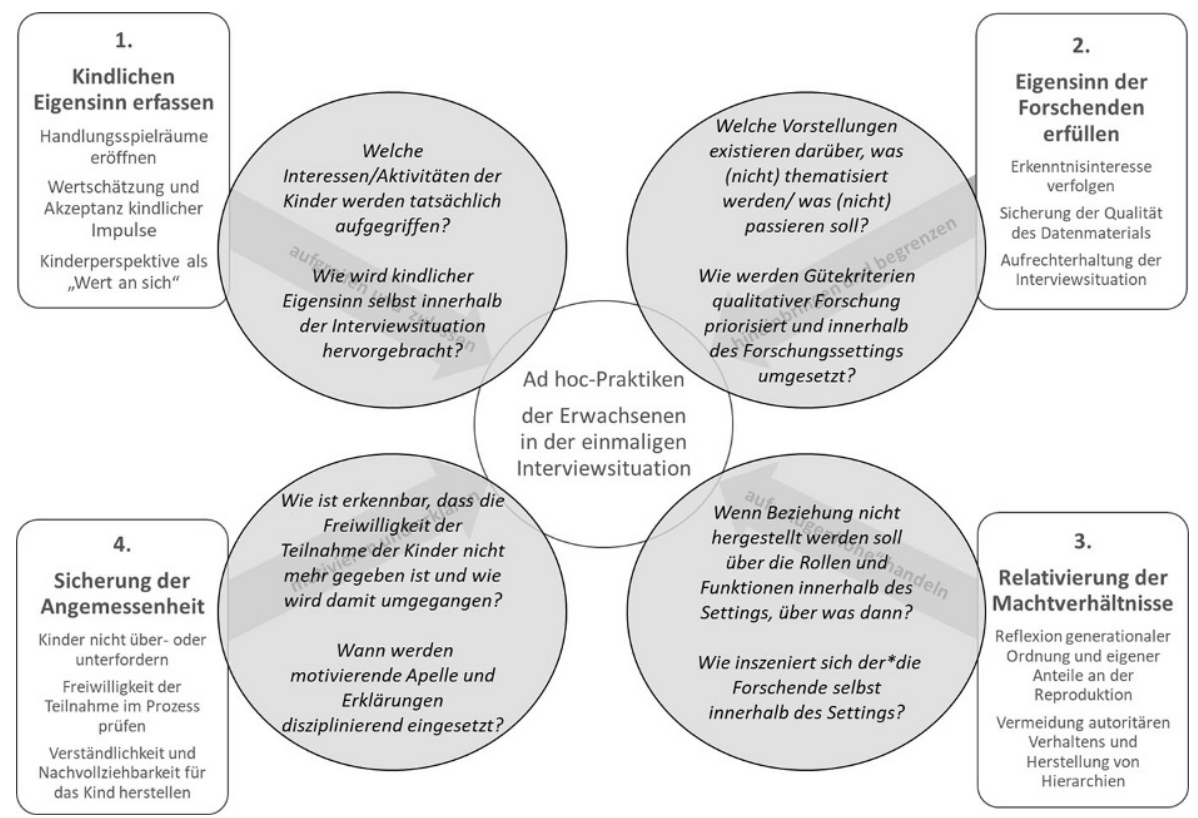

Abb. 2 Reflexionsfolien zur Erweiterung der Forschungskompetenzen Erwachsener in Befragungen mit Kindern 
Auseinandersetzung mit eigenen Normierungs- und Normalitätsperspektiven sowie Erwartungen an die Kinder vor Beginn eines konkreten Forschungsprojekts für Adhoc-Praktiken zu sensibilisieren und Handlungsstrategien für den konkreten Umgang $\mathrm{zu}$ formulieren.

Für die Weiterentwicklung von Forschungskompetenzen scheint sich das Vorgehen der Re-Analyse anzubieten, denn die rückblickende intensive Auseinandersetzung mit in ,Turning Points“ zutage tretenden ritualisierten und habitualisierten Erwachsenen-Kind-Interaktionen könnte dazu beitragen, unreflektierte Wiederholungen zu vermeiden. Besonders aus Forschungsperspektive kritische oder konflikthafte Sequenzen scheinen Potential für die Aufdeckung des beschriebenen Umschlagens der Interaktion zu haben. Ferner wäre kollegialer Austausch und die Ermöglichung von Mehrperspektivität zu empfehlen, um auch sog. blinden Flecken in Ad-hocPraktiken in der Interview-Interaktion intersubjektiv auf die Spur zu kommen. Folgende Impulse bieten sich mit Fokus auf den „Turning Point“ für den Umgang mit den formulierten vier Ansprüchen zur Gestaltung von Kinderinterviews an (Abb. 2).

So kann sich die Perspektive auf forschungsethische Anforderungen der partizipativ und inklusiv gestalteten Forschung zu den Perspektiven von Kindern hinsichtlich der Berücksichtigung von Erwachsenheit und der Reproduktion generationaler Ordnung erweitern, um das Handeln in kompetenter Gefügigkeit nicht nur von Kindern, sondern im Sinn der von Spriggs und Gillam (2017) identifizierten ethischen Herausforderung auch von Erwachsenen zu beleuchten.

Funding Open Access funding enabled and organized by Projekt DEAL.

Open Access Dieser Artikel wird unter der Creative Commons Namensnennung 4.0 International Lizenz veröffentlicht, welche die Nutzung, Vervielfältigung, Bearbeitung, Verbreitung und Wiedergabe in jeglichem Medium und Format erlaubt, sofern Sie den/die ursprünglichen Autor(en) und die Quelle ordnungsgemäß nennen, einen Link zur Creative Commons Lizenz beifügen und angeben, ob Änderungen vorgenommen wurden.

Die in diesem Artikel enthaltenen Bilder und sonstiges Drittmaterial unterliegen ebenfalls der genannten Creative Commons Lizenz, sofern sich aus der Abbildungslegende nichts anderes ergibt. Sofern das betreffende Material nicht unter der genannten Creative Commons Lizenz steht und die betreffende Handlung nicht nach gesetzlichen Vorschriften erlaubt ist, ist für die oben aufgeführten Weiterverwendungen des Materials die Einwilligung des jeweiligen Rechteinhabers einzuholen.

Weitere Details zur Lizenz entnehmen Sie bitte der Lizenzinformation auf http://creativecommons.org/ licenses/by/4.0/deed.de.

\section{Literatur}

Alderson, P., \& Morrow, V. (2020). The ethics of research with children and young people. A practical handbook (2. Aufl.). Los Angeles: SAGE.

Baader, M. S. (2018). Kinder als Akteure oder wie ist das Kind als Subjekt zu denken? Historische Kontexte, relationale Verhältnisse, pädagogische Traditionen, neue Perspektiven. In K. Koch, M. Schulz \& B. Bloch (Hrsg.), Frühpädagogische Perspektiven auf Kinder und Kindheiten (S. 22-39). Weinheim: Beltz.

Behnken, I., \& Zinnecker, J. (2001). Kinder, Kindheit, Lebensgeschichte. Seelze-Velber: Kallmeyer.

Bertram, T., Formosinho, J., Gray, C., Pascal, C., \& Whalley, M. (2016). EECERA ethical code for early childhood researchers. European Early Childhood Education Research Journal, 24(1), iii-xiii. https://doi.org/10.1080/1350293X.2016.1120533 
Boban, I., \& Hinz, A. (2009). Der Index für Inklusion. Sozial Extra, 33(9), 12-16.

Bosenius, J., \& Wedekind, H. (2004). „Mitpestümmen“. Schülerstudie 2004 des Deutschen Kinderhilfswerkes und Super RTL zur Partizipation von Viertklässlern. In D. Müller \& H. Peter (Hrsg.), Kinderreport Deutschland 2004. München: kopaed.

Bühler-Niederberger, D. (2011). Lebensphase Kindheit. Theoretische Ansätze, Akteure und Handlungsräume (Grundlagentexte Soziologie). Weinheim: Juventa.

Bühler-Niederberger, D., Gräsel, C., \& Morgenroth, S. (2015). Sozialisation 'upside down'. Wenn das Kind als Akteur die Sozialisationsperspektive erobert. ZSE : Zeitschrift für Soziologie der Erziehung und Sozialisation, 2015(2), 119-138.

Butler, M., \& Derret, S. (2014). The Walking Interview: An Ethnographic Approach to Understand Disability. The Internet Journal of Allied Health Sciences und Practice, 12(3), Article 6.

Clark, A., \& Moss, P. (2011). Listening to young children. The mosaic approach (2. Aufl.). London: ncb.

Fangmeyer, A., \& Mierendorff, J. (Hrsg.). (2017). Kindheit und Erwachsenheit in sozialwissenschaftlicher Forschung und Theoriebildung (1. Aufl.). Weinheim: Beltz Juventa.

Fuhs, B. (2012). Kinder im qualitativen Interview. Zur Erforschung subjektiver kindlicher Lebenswelten. In F. Heinzel (Hrsg.), Methoden der (2. Aufl. S. 80-103). Weinheim, Basel: Beltz Juventa.

Hansen, R., Knauer, R., \& Sturzenhecker, B. (2011). Partizipation in Kindertageseinrichtungen. So gelingt Demokratiebildung mit Kindern! Kiliansroda: das netz.

Heinzel, F., Kränzl-Nagl, R., \& Mierendorff, J. (2012). Sozialwissenschaftliche Kindheitsforschung - Annäherungen an einen komplexen Forschungsbereich. Theo-Web, 11(1), 9-37.

Hengst, H. (2017). Das Leben der Anderen. Erwachsenheit und soziologische Kindheitsforschung. In A. Fangmeyer \& J. Mierendorff (Hrsg.), Kindheit und Erwachsenheit in sozialwissenschaftlicher Forschung und Theoriebildung (S. 107-124). Weinheim: Beltz Juventa.

Höke, J. (2020). „Und die Kinderkonferenz, die haben wir abgeschafft“ Möglichkeiten kindlicher Beteiligung im Zusammenspiel von Handlungsstrategien der Erwachsenen und Kinderperspektiven in einer partizipativ arbeitenden Grundschule. DDS - Die Deutsche Schule, 112(2), 224-240. https://doi.org/ 10.31244/dds.2020.02.09

Honig, M.-S., Lange, A., \& Leu, H. R. (Hrsg.). (1999). Aus der Perspektive von Kindern? Zur Methodologie der Kindheitsforschung. Weinheim: Juventa.

Kasüschke, D. (2016). Kinderstärkende Pädagogik und Didaktik in der KiTa. Stuttgart: Kohlhammer.

Kelle, H., \& Schweda-Möller, A. (2017). Weder Reifizierung noch Negierung der Differenz Kinder - Erwachsene. Über ethnographische Gratwanderungen und ihre methodologische Reflexion. In A. Fangmeyer \& J. Mierendorff (Hrsg.), Kindheit und Erwachsenheit in sozialwissenschaftlicher Forschung und Theoriebildung (S. 125-140). Weinheim: Beltz Juventa.

Lundy, L. (2007). 'Voice' is not enough: conceptualising Article 12 of the United Nations Convention on the Rights of the Child. British Educational Research Journal, 33(6), 927-942.

Schütz, A., Breuer, A., \& Reh, S. (2012). Sequenzanalsysen von Kinder-Interaktionen. Zu den Möglichkeiten einer sozialwissenschaftlichen Hermeneutik. In F. Heinzel (Hrsg.), Methoden der Kindheitsforschung (2. Aufl. S. 190-204). Weinheim, Basel: Beltz Juventa.

Spriggs, M., \& Gillam, L. (2017). Ethical complexities in child co-research. Research Ethics, (1), 1-16. https://doi.org/10.1177/1747016117750207

Velten, K. (2019). HandlungsSpielRäume - Selbstwirksamkeitserfahrungen von Kindern in Kindertageseinrichtung und Grundschule. Zeitschrift für Grundschulforschung (ZfG), 12(1), 165-179.

Wöhrer, V., Arztmann, D., Wintersteller, T., Harrasser, D., \& Schneider, K. (2017). Partizipative Aktionsforschung mit Kindern und Jugendlichen. Wiesbaden: Springer.

World Vision e.V. (Hrsg.). (2018). Was ist los in unserer Welt? Kinder in Deutschland, Bd. 4. Weinheim/ Basel: Beltz. 